\title{
Increased Sacral Uptake on a Bone Scan with SPECT/CT in a Patient with Achondroplasia: Normal or Abnormal?*
}

Alexander Copelan ${ }^{1}$, Kristin Booth $^{2}$, and Helena Balon ${ }^{1}$

${ }^{1}$ Beaumont Health System, Royal Oak, Michigan; and ${ }^{2}$ McLaren Oakland Medical Center, Pontiac, Michigan

\begin{abstract}
A additional SPECT/CT imaging of the pelvis was performed to clarify the findings. SPECT data.

\section{QUESTION 1} the planar images?

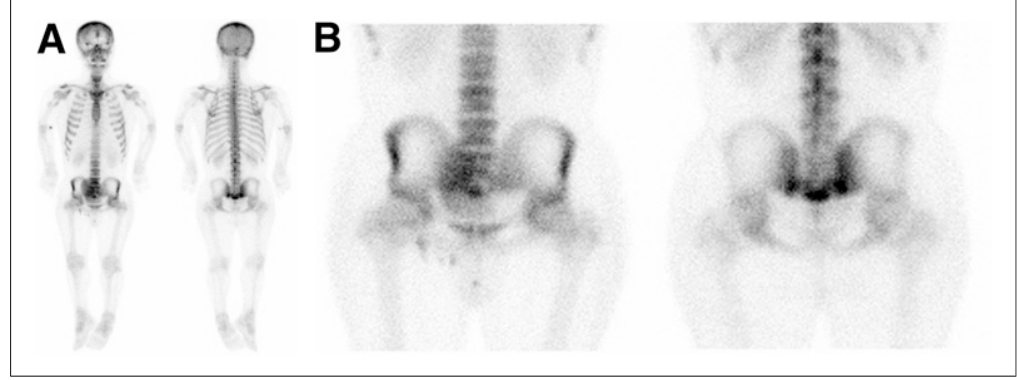

26-y-old man with achondroplasia presented with chronic lower back pain with no history of trauma. MR imaging performed 5 mo previously demonstrated a congenitally small lumbar canal causing multilevel stenosis.

After intravenous administration of $814 \mathrm{MBq}(22 \mathrm{mCi})$ of ${ }^{99 \mathrm{~m}} \mathrm{Tc}-$ methylene diphosphonate, delayed anterior and posterior whole-body bone scans were performed, along with spot views of the pelvis. On review of the planar images,

Limited-field-of-view, low-dose CT imaging was performed for anatomic localization and attenuation correction of the

After reviewing Figures 1-4, what is the most likely cause of the focal increased uptake in the sacral region seen only on

FIGURE 1. Whole-body bone scan and spot views of pelvis demonstrate mild to moderate increased radiotracer activity in right and left sacroiliac joints inferiorly and in sacrococcygeal area, raising possibility of fracture. Mild focal increased uptake is also noted bilaterally along proximal humeral cortices.

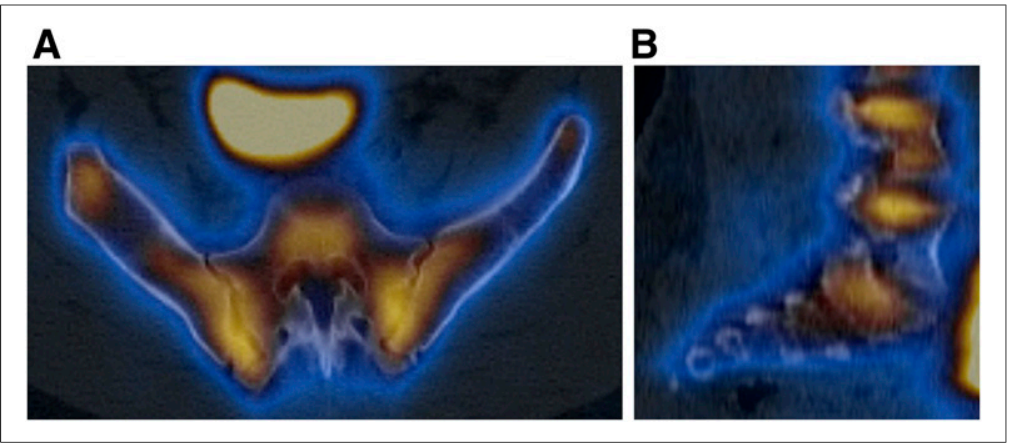

FIGURE 2. Axial (A) and sagittal (B) SPECT/CT images of pelvis and sacroiliac joints demonstrate mild increased radiotracer activity in sacroiliac joints bilaterally. Sacrococcygeal uptake seen on planar images is not apparent on SPECT images.
FIGURE 3.

Champagne glass appearance of pelvis (arrow), which is due to anteversion of pelvis, squaring-off of ilia, lower lying anterior superior iliac spine, and higher posterior superior iliac spine.

FIGURE 4.

Sagittal slice (from SPECT/CT) demonstrating horizontal configuration of sacrum.
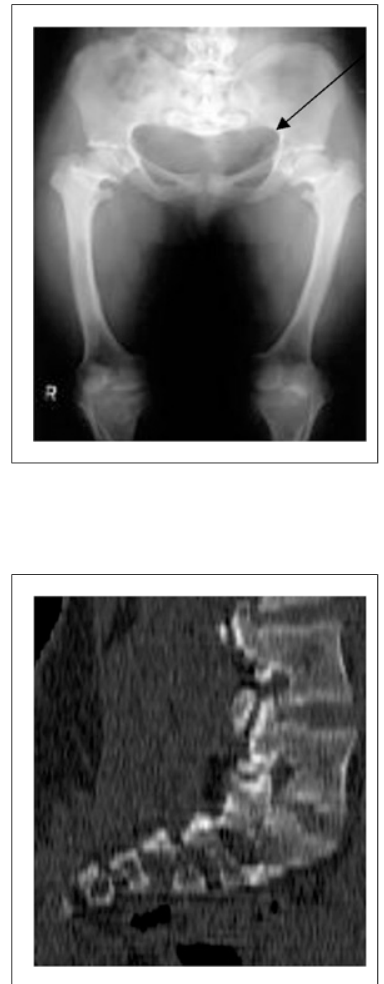

A. Sacral fracture.
B. Osteomyelitis.
C. Horizontal orientation of the sacrum.
D. Osteoid osteoma.

\section{QUESTION 2}

Which of the following is true regarding achondroplasia?

A. It can be diagnosed on prenatal ultrasound.
B. It is the second most common cause of dwarfism.
C. It is typically diagnosed with genetic testing.
D. It affects the axial skeleton more than the appendicular skeleton.

\section{QUESTION 3}

Which of the following is not associated with achondroplasia?
A. Narrowing of the spinal canal.
B. Below average IQ.
C. "Champagne glass" pelvis.
D. Depressed nasal bridge

\section{QUESTION 4}

Which of the following is a major complication later in life in achondroplasia patients?

a. Genu valgus of the knee.

b. Pulmonary hypertension.

c. Infertility.

d. Foramen magnum stenosis.

\section{DISCUSSION}

Achondroplasia is the most common cause of dwarfism. About $75 \%$ of cases are due to sporadic mutations, often associated with advanced maternal age, but it can also be inherited as an autosomal dominant genetic disorder. Achondroplasia occurs because of an abnormality of cartilage formation secondary to a mutation in the gene for fibroblast growth factor receptor 3 (1). The condition can be diagnosed with prenatal ultrasound or on the basis of clinical and radiographic findings; genetic testing is not typically needed (2). The main radiographic findings involve the skull, extremities, spine, and pelvis. Narrowing of the spinal canal is the pathologic hallmark (3). Platyspondyly (flattened vertebral bodies) can be seen, and decreasing interpedicular spaces caudally from L1 to L5 can lead to spinal stenosis and lower back pain. The skull often demonstrates a prominent forehead and depressed nasal bridge. The skull base is disproportionately small because it forms by endochondral ossification, whereas the skull vault is normal because it forms by membranous ossification. This disproportionate growth can lead to a small cisterna magna, empty sella, and a narrowed foramen magnum, which may necessitate cervicomedullary decompression $(4,5)$. The extremities are disproportionately shortened, especially proximally-known as rhizomelic shortening - and are often widened because of unopposed appositional bone growth. This is the most likely explanation for the faint uptake in the proximal humeri in Figure 1. The pelvis can demonstrate a champagne glass appearance secondary to anteversion, squared-off and shortened ilia, a low anterior superior iliac spine, and a high posterior superior iliac spine. The pelvis itself is typically small, and horizontally oriented acetabula are frequently seen.

\section{REFERENCES}

1. Horton WA, Hall JG, Hecht JT. Achondroplasia. Lancet. 2007;370:162-172.

2. Modaff P, Horton VK, Pauli RM. Errors in the prenatal diagnosis of children with achondroplasia. Prenat Diagn. 1996;16:525-530.

3. Lonstein JE. Treatment of kyphosis and lumbar stenosis in achondroplasia. Basic Life Sci. 1988;48:283-292.

4. Smoker WR, Khanna G. Imaging the craniocervical junction. Childs Nerv Syst. 2008;24:1123-1145.

5. Morgan DF, Young RF. Spinal neurological complications of achondroplasia: results of surgical treatment. J Neurosurg. 1980;52:463-472.

${ }^{\star}$ For the answers, see page 241. 